Remarquons que :

$$
b=\frac{a}{\Omega}=\frac{R_{2}}{L_{2} \Omega}
$$

II. Caractérislique mécanique rapportée à $\omega^{\prime}$. - Un changement intuilif d'axes donne celle nouvelle caractéristique mécanique.

(A suivre.)

L. Barbillion et F. Glaret.

\section{ÉCONOMIE INDUSTRIELLE}

\section{LES TURBINES A VAPEUR}

\section{DESCRIPTION ET CLASSIFICATION}

A la fin de l'année rgro, on pouvait compler comme turbines à vapeur en fonclionnement : près de $x$ ooo turbines Rateau ou du genre Rateau, telles que les turbines Oerlikon et les turbines Zoelly ; presque autant de turbines de la Société d'électricité A. E. G. de Berlin; el un nombre un peu plus grand de turbines Brown-Boveri-Parsons.

Le nombre total des turbines, établies suivant le système Parsons, dépassait à cette date 2500 unités.

Sans compter les turbines de Laval, très répandues aclue]lement mais ne représentant que des machines d'assez faible puissance, on peut dire qu'il est déjà sorti des usines de construction de l'Europe seulement plus de 5 ooo turbines à vapeur.

A ce chiffre, il faudrait ajouter les turbines marines qui sont déjà très répandues puisque, pour le système Parsons sculement, la puissance des turbines, employées à la propulsion des navires, dépasse actuellement 4300 ooo HP.

Ces chiffres sont assez éloquents par eux-mêmes et montrent suffisamment le développement considérable pris ces dernières années par les turbines à vapeur.

Presque toutes ces machines sont de grande puissance et la plupart peuvent donner plusieurs milliers de chevaux. Les turbines de to ooo HP sont acluellement de construction courante et celles de 20000 HP ne sont pas rares. Nous avons eu l'occasion, l'année passée, d'en voir quelques-unes en montage dans les ateliers de la Société A.E. G., à Berlin. Elles étaient destinées à une grande Centrale du Sud de l'Afrique qui devait recevoir sept unités de $20000 \mathrm{HP}$ pour fournir la force motrice à toutes les mines du Transvaal. Au moment de notre visite, les Ateliers de l'A. E. G. avaient à l'étude une lurbine de 30 ooo HP pour une puissante Centrale de Berlin.

Cependant, toutes Ics turbincs Rateau, Zolly, BrownBorcri, A. E. G., Curtis, ctc., sont postérieures à I goo. Avant cette date, on ne connaissail guère que les turbines Parsons ct colles de Laval.

La Maison Saulter flarlé a commencé la construction des turbines Raleau vers $1897^{-1} 898$ el les premières turbines essayées onl élé des machines marines. Mais ce n'est qu'en rgo2 que M. Raleau a mis en marche, aux Mines de Bruay, sa première turbine à terre, qui est restée célèbre parce qu'elle étail également la première ulilisant la vapeur d'échappement d'une machine à piston. Le fonctionnement. de cetle turbine ful d'ailleurs des plus satisfaisants.

Au commeneement de rgo5 seulement, sortirent des Ateliers Escher Wyss, à Zurich, les premières turbines étudiées par M. Zoelly, directeur de ces Ateliers. Ces lurbines, qui sont en principe semblables à celles de M. Rateau; se sont répandues rapidement grâce à leur excellente fabrication, Elles sont actuellement construites en France par MM. Schneider et $\mathrm{C}^{10}$, au Creusol, la Société Alsacienne de Constructions Mécaniques et la Société de l'Horme et de la Buire.

MM. Brown-Boveri et $\mathrm{C}^{\mathrm{io}} \mathrm{n}$ 'ont obtenu la licence, pour la construction des turbines Parsons, qu'en rgoo, et les premières turbines construites à Baden datent du début de Igor.

Enfin, la Société d'Electricité A. E. G., de Berlin, ne commença à s'occuper des turbines à vapeur que vers r go2-1 g03 et construisit d'abord les turbines des professeurs Riedler et Stumpf qu'elle ne tarda pas à abandonner pour adopter exclusivement son type actuel qui date de rgo4. C'est pendant celle même année qu'elle acheva, à Berlin, l'aménagement de sa grande fabrique de turbines qui occupe actuellement plus de 3500 ouvriers travaillant jour et nuit en trois équipes.

Depuis longtemps, les inventeurs ont étudié les turbines à vapeur, et en parliculier, dès 1853 , l'ingénieur français Tournaire donnait d'une façon très précise les règles à suivre pour élablir une turbine à réaction; mais ce sont surtoul quatre ingénieurs, dont il est à peine besoin de rappeler ici les noms: Parsons, de Laval, Rateau et Curtis, qui ont contribué à rendre ces machines vraiment industrielles.

Les difficultés à surmonter ont été considérables et, malgré tout le talent et la ténacité des inventeurs précédents, il n'aurait pas été possible de parvenir aux résultats actuels sans les progrès récents de la mécanique et surtout de la métallurgie.

ll est bon d'observer aussi que le succès des turbines à vapeur est dû en grande partie à l'électricité et que, sans elle, les applicalions des turbines auraient été assez resIreintes, surtout pour les grandes puissances. Il est juste de remarquer également que les électriciens ont eu à résoudre alors des problèmes au moins aussi difficiles que ceux que les constructeurs de turbines sont parvenus à solutionner.

Pour les turbo-alternateurs et surtout pour les turbodynamos, les électriciens, en effet, ont eu bien souvent, c'est le cas de le dire, du fil à retordre. Cela se conçoit facilement. Les machines électriques sont composées des matières les plus diverses : acier, cuivre, isolant, et leur manque d'homogénéité donne lieu à des difficultés certainement beau* coup plus grandes, à cause de la force centrifuge, que dans le cas des turbines à vapeur. Si l'on ajoute à cela que ces machines, devant fournir une très grande puissance sous un faible volume, chauffenl souvent beaucoup plus qu'on ne le voudrail ct que les échauffements successifs délerminent des déformations que rendent difficiles à éviter le manque d'homogénéité de l'ensemble et surtout la présence des isolants, on s'explique alors facilement pourquoi les éclatements des machines électriques ont été plus fréquents que ceux des turbines et pourquoi ces éclatements sont actuellement encore plus à redouter pour les alternateurs et les dynamos que pour les turbines. Aussi, ne peut-on que trouver très justifiée la sage précaution, prise par certains constructeurs comme la société A. E. G. et la Maison Sicmens Schuckert, d'installer des locaux spéciaux, ordinairement souterrains et garnis de solides madriers, dans lesquels les machines essayées à l'emballement peuvent éclater toul à leur aise sans gêner personne.

Actuellement, les turbo-alternateurs sont construits d'une façon courante par un grand nombre de maisons el l'on parvient normalement à établir des machines très puissantes tournant à très grande vitesse. Il est possible, par exemple, de faire des turbo-alternateurs de 3 ooo et même 3500 kilo- 
volls-ampères, tournant à 3 ooo tours par minute, ou bien de $7500 \mathrm{~K}$. V. A. fonclionnant à une vitesse de I 500 tours.

Ouant aux turbo-dynamos, les difficultés rencontrées sont encore plus considérables et c'est à peinc si actucllement (a) Europe quatre ou cing constructeurs peuvent montrer plusieur's de ces machines en fonctionnement satisfaisant.

Dans les turbines à vapeur, la source des principales difficultés provienl de la vitesse énorme prise par la vapeur cn se détendant.

De la vapcur à ro $\mathrm{kgs}$ de pression, surchauffée à $250^{\circ}$, sécoulant par une tuyère dans un condenseur où l'on peut très facilement oblenir une pression absolue de o, I $\mathrm{kg}$., arquicht une vitesse de I 200 mètres par seconde.

Les vitesses considérables de la vapeur exigent des roues founnanl avec une très grande rapidité et donnant lieu à une puissance perdue par frottement imporlanle puisque celle jerte est proportionnelle au cube de la vitesse de la vapeur.

\section{Turbines à action}

\section{Turbines à plasieurs étages de vilesse}

Pour utiliser l'énergie cinćlique de la vapeur, on peul l'employer qu'une seule roue, comme dans les turbines de

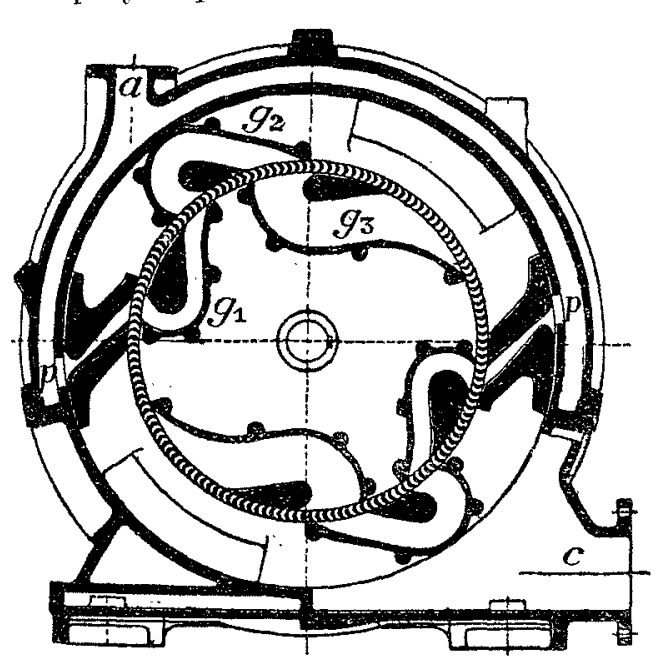

Fig. I. - Turbine Electra

a. Arrivée de la vapeur.

p. Tuyères pour la détente de la vapeur.

$g_{1}, g_{2}, g_{3}$ Clarinettes ramenant la vapeur sur la rone

c. Ecrappement au condenseur.

lement obliger la vapeur à revenir plusieurs fois sur la même roue comme dans les turbines Riedler et Stumpf, construites aulrefois par l'A. E. G., ou comme dans la turbine Electra, imaginćc par l'ingénieur Kolb, de Karlsruhe, et conslruite en France par la Compagnie Générale Electrique de Nancy. Dans celle lurbine, le retour de vapeur se fait au moyen de pièces lixes en forme d'U appelées clarinetles, qui ramènonl jusqu'à trois fois la vapeur sur la mème roue.

De telles lurbines sont dites à élages de vilesse.

\section{Turbines à plusieurs étages de pression}

Mais les grandes vitesses de vapeur ne sont admissibles que pour les faibles puissances parce qu'elles conduisent à de trop fortes consommations de vapeur à cause des pertes par frottement.

Lorsqu'on aborde les grosses unités, pour pouvoir lutter avec les machines à piston si perfectionnées à l'heure acluclle, on est obligé de limiter la vitesse de la vapeur et, pour cela, de fairc des délentes successives.

On est donc conduit à employer en quelque sorte plusieurs turbines élémentaires calées sur le même arbre, ou même séparées par groupe, et n'utilisant chacune qu'une pelite chute de pression.

Ainsi, au-dessus de roo IIP et jusqu'à 3oo IIP, la Maison Breguet, qui construil la turbine de Laval, divise la chule de pression en deux parties ef fait agir la vapeur sur deux roues différentes. La première roue, par cxemple, utilisera une chute de pression de $\mathrm{x} 2 \mathrm{kgr}$. à $2 \mathrm{kgr}$. et la seconde de 2 kgr. à la pression du condenseur. 11 en est de même des turbines Electra entre 5o et $r$ ooo HP ol des turbines de moyenne puissance des Alelicrs Oerlikon, de la Société A. E. G., etc...

Mais deux chules de pression ne sont admissibles que pour les moyemnes puissances, et au-delà i] est indispensable de diviser davantago la détente de la vapeur el d'augmenter' ce que l'on appelle les ćlages de pression, d'autani plus que, si l'on admet pour chafue turbine ćlémentaire la même puissance à produire, la vilesse de la vapeur ne se lrouve pas divisée par le nombre d'étages de pression, mais par la racine carréc de ce nombre d'élages.

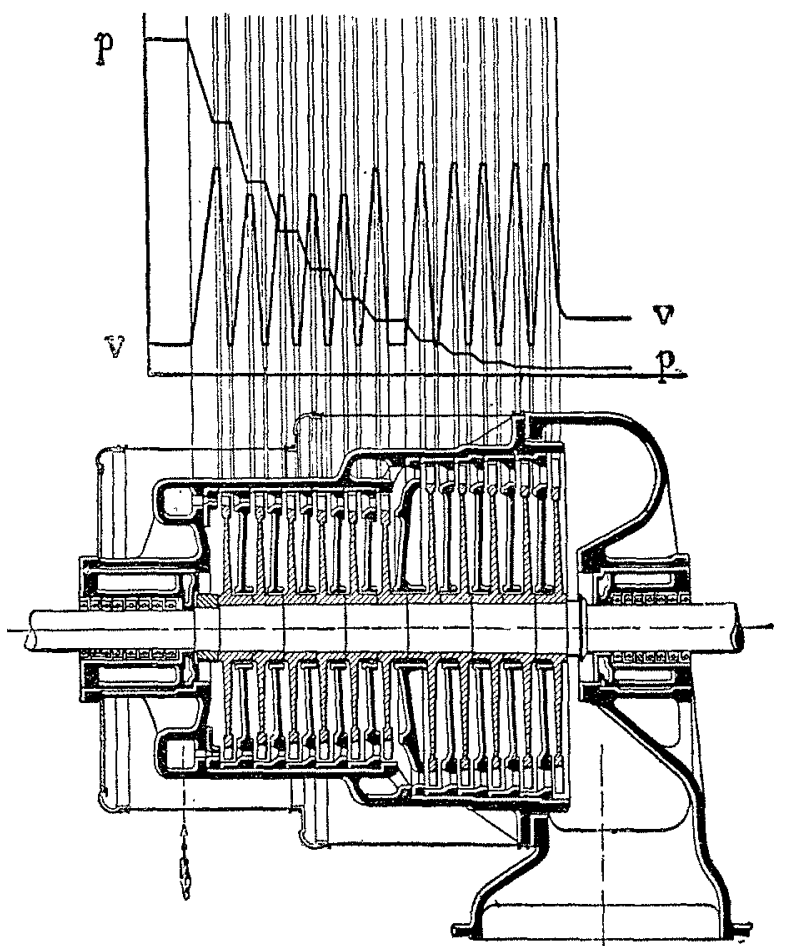

FIg. 2. - Turbine Rateau

p. Pression de la vapeur dans les différentes parties de la turbine. v. Vitesse de la vapeur.

Ainsi, en adoptant 9 élages de pression, pour passer de Io $\mathrm{kgr}$. à o, r $\mathrm{kgr}$., la vitesse de la vapeur, au lieu d'être de I 200 mèlres comme pour une détente unique, serait environ de s soo: $V \overline{9}=$ foo mètes pour chaque étage.

Dans les turbines Raleau et Zoelly, par excmple, les vilesses de vapeur ne dépassent pas en général 350 à $400 \mathrm{~m}$.

On voit done que si l'on veut oblenir des vilesses de vapeur relativement faibles, il est nécessaire d'augmenter beaucoup le nombre d'étages de pression. Ce nombre d'étages sera d'autant plus grand que la puissance sera plus considérable. Ainsi, les turbines Zoelly possedent ordinairement :

so étages de pression de 200 à I 300 HP pour 3 ooo tours, r6 - - jusqu'à $5000 \mathrm{HP}$ - $1500-$ 20 - de 5000 à $8000 \mathrm{HP}-\mathrm{x} 000-$

A chaque turbine élémentaire, c'està-dire à chaque étage. de pression, pourra ne correspondre qu'un seul étage de vitesse si l'écoulement de la vapcur n'est pas trop rapide par suite de l'adoplion d'un grand nombre d'étages de pression. 
C'est le cas de la turbine Rateau et des turbines semblables Oerlikon et Zoelly.

Mais si l'on veul, pour simplifier la turbine, n'admettre qu'un pelit nombre d'élages de pression, on cst alors obligé, pour ne pas avoir de trop grandes vitesses de rotation, d'employer plusieurs élages de vitesse par chuie de pression. C'est ce qui a lieu pour les turbines Curtis, construites principalement en Amérique par la General Electric Company et en France par la Compagnie Thomson-Houston.

Il est prudent, cependant, de ne pas trop réduire le nombre des étages de pression parce que les consommations de vapeur peuvent devenir cxagérées; bien des constructeurs

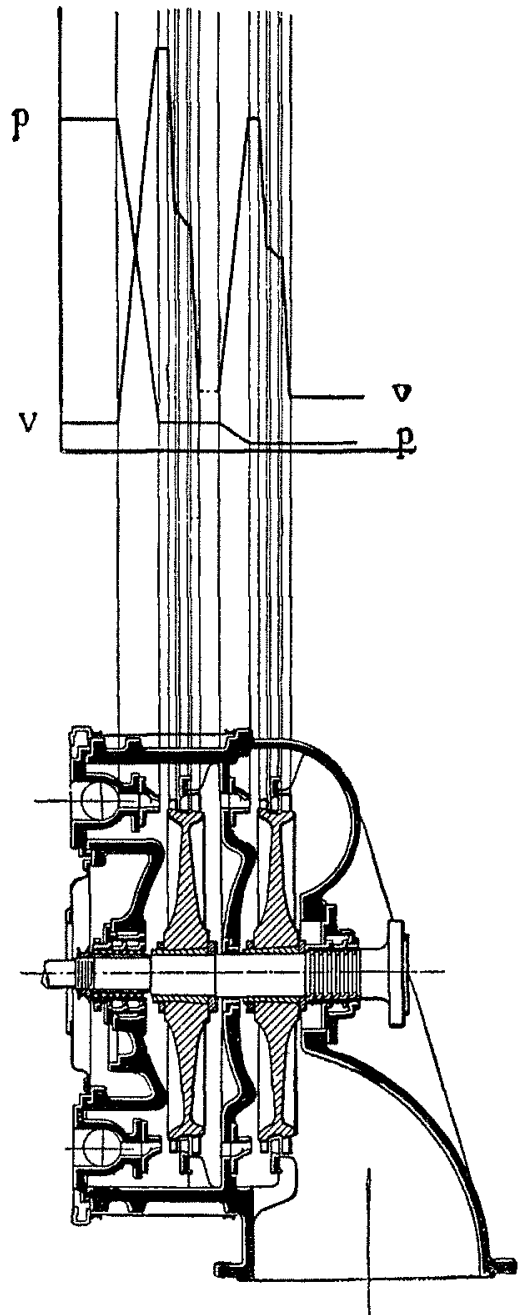

Fig. 3. - Turbine du Type Curlis

A 2 étages de pression et à 2 étages de vitesse par étage de pression.

$p$. Pression de la vapeur.

v. Vitesse de la vapeur. pression el se rapproche de la lurbine Ralcau pour la pautie basse pression.

\section{Inconvènient des turbines à plusieurs chutes de pression Turbines à disques et turbines à tambour.}

En employant ainsi plusieurs petites turbines élémentaires, correspondant chacune à une chute de pression, on gagne bien l'avantage de réduire considérablement la vitesse de la vapeur el, par suite, les pertes par frottement, mais cet avantage est compensé, en partic, par quelques inconvénients.

Tout d'abord, la machine est plus compliquée et plus coûteuse puisque, au lieu de ne faire qu'une seule roue, on doit en réalité construire plusieurs petiles turbines.

D'autre part, la pression étant différente dans chaque pe- tite turbine élémentaire, on est obligé de prévoir des presseétoupes à chaque étage de pression pour éviter les fuites de vapeur d'une turbine dans l'aulre, fuites qui peuvent augmenter considérablement la consommation de vapeur.

Dans les turbines du genre Rateau, plusieurs disques munis d'ailettes sont calés sur le même arbre et correspondent chacun à un étage de pression. Entre ces disques mobiles se trouvent des disques fixes ou diaphragmes en fonte ou en acier à la périphéric desquels sont percés des orifices pour faire écouler la vapeur et former distributeur. Ces diaphragmes entourent l'arbre avec un jeu très faible et des canelures de façon à jouer le rôle de véritables presse-étouppes

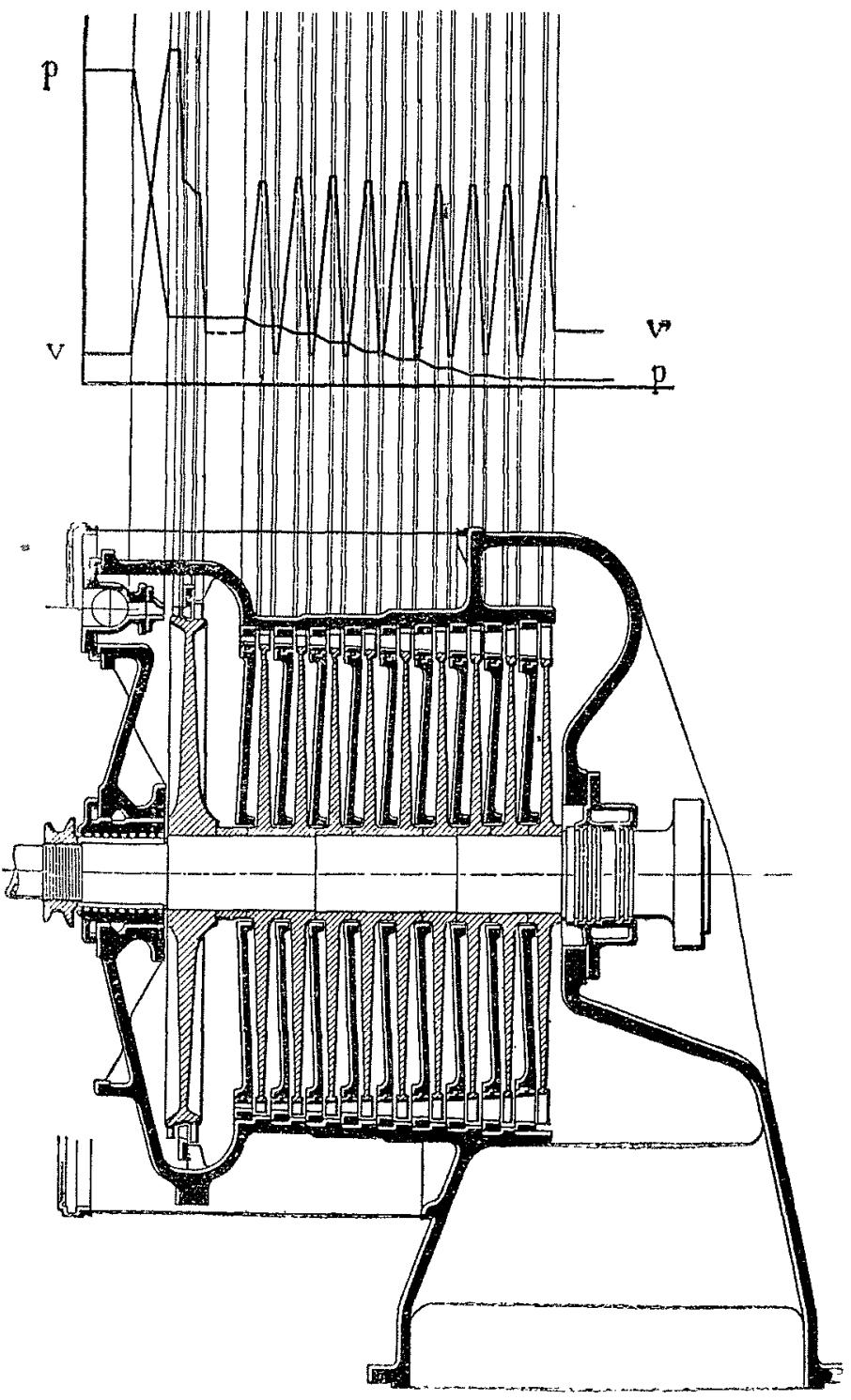

F1G. 4. - Turbine A.E.G.

$p$. Pression de la vapeur. - $v$. Vitesse de la vapeur.

mais sims partic frollanle. Finlre les deux faces de ces disques, ì cause de la détente de la vapeur dans lo distributeur, il existe une différence de pression qui délermine des efforts considérables pouvanl fairc voiler ces disques el qui donne lieu à des fuites de vapeur au passage de l'arbre.

Avec l'emploi d'un très grand nombre d'étages de prossion, on a également l'inconvénient d'augmenter les surfaces de froltement de la vapeur et, si les pertes dues au frottemenl de la vapeur dépendent de la vitesse, elles sont aussi proportionnelles à la surface. En oulre, les disques tournant dans la vapeur, donnent licu à des pertes par frottement très sensibles et ces pertes qui sont proportionnelles au poids spécifique de la vapeur sont d'autant plus importantes que la pression est plus élevée. 
Afin de réduire ces pertes el aussi pour simplifier la construction des turbines, on a proposé de disposer toules les aubes fixes et mobiles sur deux cylindres concentriques. Gest pour ces raisons que les Maisons Breguet et Dujardin de Lille ont adopté cclte disposition. Leurs turbines sont du genre Rateau, c'est-à-dire à étages de pression avec un scul étage de vitesse par étage de pression, mais les disques mobiles sont remplacés par un tambour muni d'ailettes lournant à l'intérieur d'un tambour fixe portant également des aileltes destinées à former distributeur.

Si l'on peul éviter ainsi les inconvénient des disques, l'on a par contre d'autres désavantages. D'abord les fuites de vapeur sont relativement beaucoup plus importanles parce que, an lieu de se produire autour de l'arbre, elles ont lieu i la surface d'un tambour, c'est-à-dire sur une plus grande

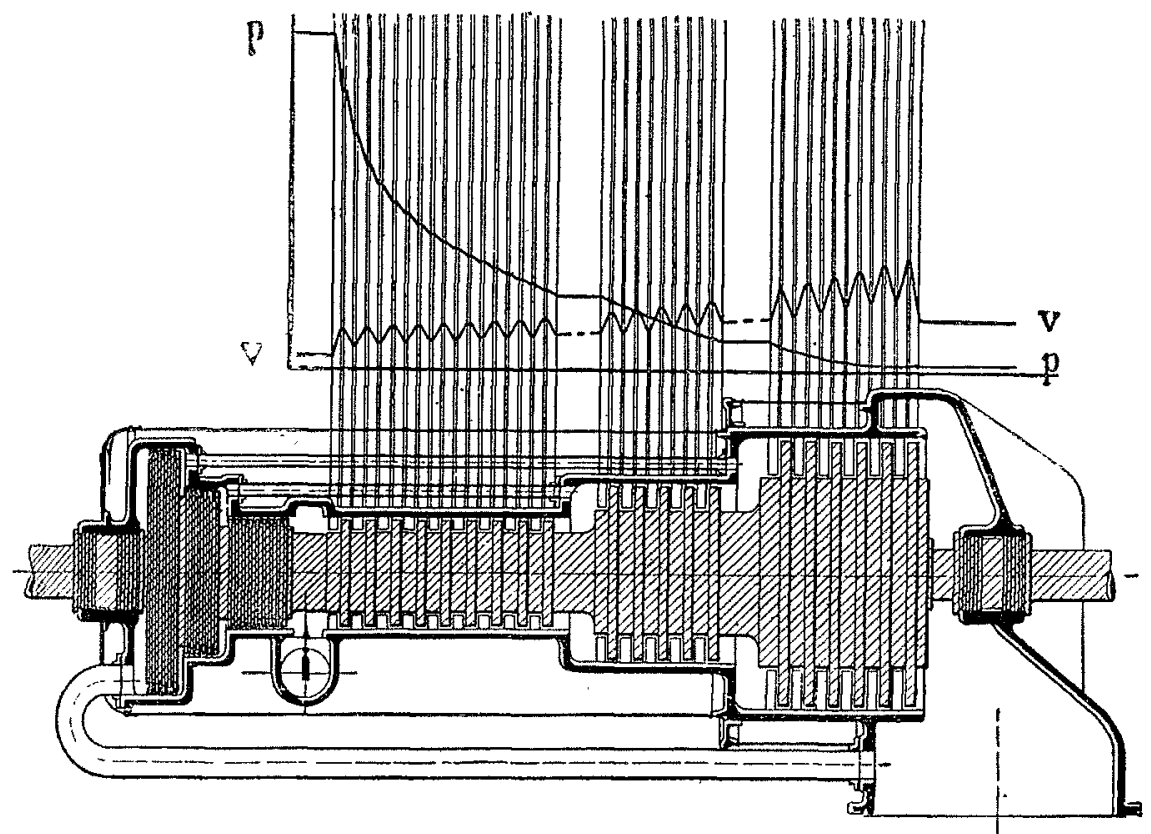

Fig. 5. - Turbine Parsons

$p$. Pression de la vapeur $-v$. Vitesse de la vapeur.

rirconférence. Pour réduire ces fuiles, on esi obligé d'adopar un nombre d'étages de pression relativement plus considérable que dans le cas des turbines à disques. D'ailleurs, les constructcurs des turbines à tambour cmploicnt aussi parfois à l'entréc de leur turbine, dans la partic à hautc prossion, le système à disques lorsque, le débit de la vapeur ćant faible, il est nécessaire de réduirc les fuiles au mininum nossible.

D'autre part, comme la vapeur au débul de la lurbine est sensiblement à la pression de la chaudière, fandis qu'à l'extrémilé olle se lrouve être à la pression du condenseur, i] existe done entre les deux faces du tambour une très grande différence de pression produisant, suivant l'arbre, une forte pousséc qu'il faut pouvoir équilibrer. Celle poussée peut l'ailleurs, dans certains cas, être avantageuse, comme par cxemple dans les turbines marines où elle esl utilisće à contrebalancer la réaction des hélices ; aussi, beaucoup de constructeurs adoptent-ils la forme en lambour pour la partic basse pression de leurs turbines marines.

Dans toutes les turbines précédentes, la vapeur agit par impulsion. Les chutes de pression se produisent entièrement dans des distributeurs fixes d'où la vapeur s'échappe avec toule sa vitesse pour venir frapper les aubes mobiles qui tournent dans un milieu à pression constante.

Ces turbines sont dites $\grave{a}$ action.

\section{Turbines à réaction}

Dans le bul de réduire cncore davantage la vilesse de la vapeur afin de diminuer les perles par frollement el supprimer toute usure des aubes, on peul, au lieu de faire détendre la vapeur par étages dans les distributeurs sculement, admethe également une chute de pression dans les aubes mobiles et réaliser ainsi dans loute la turbine une détente progressive et continue de la vapeur depuis la chaudière jusqu'au condenseur.

En se détendant dans les aubages mobiles, la vapeur agil alors par réaclion. Parsons, on le sail, est le premier qui soit parvenu, après bien des efforts, à mellre au point la turbine à réaction qu'il éludiait depuis 1884. Il a créć, pour cela, des dispositifs qui sont reslés classiques.

Avec le système à réaction, la vilesse de la vapeur peut être très réduile et, dans les lurbines Parsons, elle ne dépasse pas ordinairement 100 mètres par seconde; la vilesse langenlielle des aubes mobiles peut donc être relativement faible. Par contre, on se trouve en présence de l'ès grosses difficultés provenant surtoul de ce que, la délente de la vapeur se faisanl dans les aubes mobiles, il cxiste une différence de pression entre les deux rotés de ces aubes of il en résulle: Une ponssíte surVANT L'ARBRE; Des FutTes de valeur so produisanl enle les aubes mobiles el la parlic lixe ; L'onturaTHON D'EMPLOYER h'INAGTON TOTAL pour éviler les pertes hop grandes qui se produinatonl incivilablement dans les aubes mobiles avere l'injecliont parliclle par suite des fuiles of du lombillonnemont de la vapeur.

la poussée suivanl l'arbre, qui d́lail an débul un des gros inconvénients des lubbines à róaclion, est à l'heure actuelle facilement contrebalaneco soit pal les pistons équilibreurs bien connus, imaginés par Parsons (fig. 5), soil plutol mainlenant par l'emploi de lubines doubles (ligr. 7 of 8), on par l'inversion du sens d'écoulement de la vapeur lor'syue colle-ci passe a la partie basse pression (fig. (6), ou par la disposition du limbour mobile qui est préva de telle

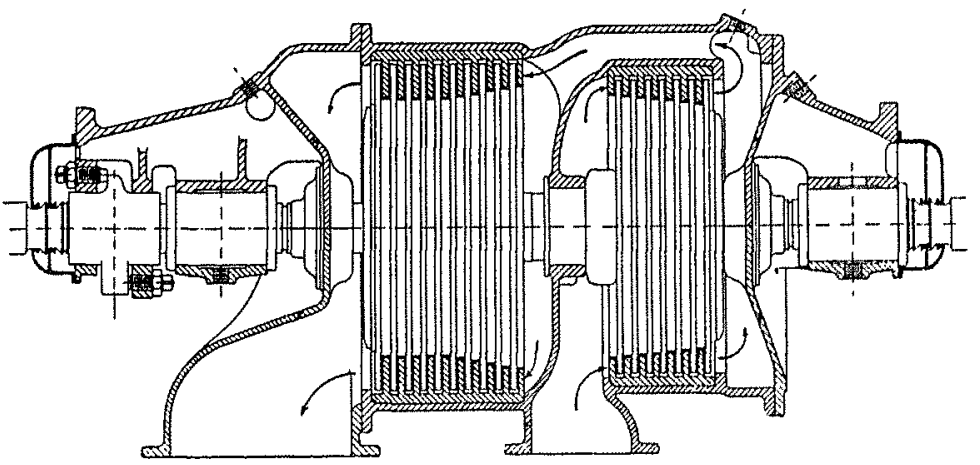

Fig. 6. - Turbine à lambour Bréguel

Avec inversion du sens d'ćcoulement de la vapeur.

façon que la vapeur, agissant sur le fond de ce lambour, puisse équilibrer la pousséc de la vapeur sur les ailelles (Brown-Boveri), ou au moyen d'un piston contre lequel agit de l'huile sous pression (Sulzer), ou enfin par un simple palier de butée qui peut être suffisant dans les lurbines mixles ou combinées.

De même que pour les turbines à action, la poussée suivant l'arbre peut être utilisée pour équilibrer la réaction des hélices et la Société A. E. G., par exemple, emploic 
exceptionnellement dans ce but le tambour à réaction pour la partie basse pression de ses turbines marines.

Les fuiles de vapeur avec les turbines à réaction se produisent aussi bien entre les arbres mobiles et le cylindre fixe que dans le distribulcur entre les ailettes fixes et le tambour mobile. Ces fuiles dépendent de la scclion offerte inutilement au passage de la vapeur, du rapport des pressions existant à l'entrće et à la sortic des aubes fixes ou mobiles et aussi du poids spécilique de la vapeur, de sorte qu'elles sont relativement moins importantes dans la partic basse pression de la turbine que dans la partie haule pression.

Pour réduire la section de passage correspondant aux fuiles, on a été amené à n'admettre qu'un jeu très faible, ne dépassant pas parfois $4 / \mathrm{IO}^{\circ}$ à $5 / 10^{\circ}$ de millimètre entre la parlie fixe et la partie mobile. Il en est résulté, trop souvent, des contacts entre le rotor el le stator déterminant ce que l'on appelle une salade d'ailettes.

Afin de diminuer le plus possible le rapport des pressions, on a dû adopter un très grand nombre d'étages de pression. Les turbines Parsons renferment souvent plus de $\mathrm{I} 60 \mathrm{ran}$ gées d'aubes fixes et mobiles disposées les unes à la suite des autres et représentant autant de chutes ou d'étages de pression. Avec un aussi grand nombre de rangćes d'aubes, on ne peut songer évidemment à adopter les disques mobiles et les diaphragmes fixes des turbines du genre Rateau, aussi l'emploi du tambour est-il dans ce cas obligatoire.

L'imporlance des fuites dans les turbines Parsons a été exagérée et d'ailleurs celles-ci cxistent aussi dans les turbines à action. Avec les turbines mixtes ou combinćes actuelles, les jeux entre les parties fixes el mobiles pouvant être suffisamment grands, les salades d'ailettes ne sont pour ainsi dire plus à craindre.

L'obligation de faire l'injection tolale constitue certainement le plus grave inconvénient des turbines à réaction. Dans le cas, en effet, des faibles puissances, pour la partic haute pression, la vapour n'exigeant qu'une seclion d'écoulement relativement très réduite; on cst d'abord obligé de diminuer énormément la hauleur des aubes qui n'atteignent plus que quelques millimètres, ce qui, même avcc de très faibles jeux, conduit à des fuites de vapeur relativement importantes, ensuite il est indispensable de n'adopler pour le tambour qu'un diamètre très petit ; il en rćsulte une faible vitesse tangentielle et par suite l'obligation d'admeltre un grand nombre d'étages de pression pour que la vilesse de la vapeur ne soit pas trop grande par rapport à la vilesse tangenticlle. La partie à haute pression de la turbine ne fournit done que peu de puissance el dans des conditions assez mauvaises à cause de l'imporlance des fuiles et des pertes par frottement.

\section{Turbines mixtes ou combinées à action et à réaction}

La turbine à action, avec l'injection partielle est évidemment bien supérieure dans ce cas; aussi les constructeurs de la lurbine Parsons, notamment la Sociélé Westinghouse d'Amérique, la première dès Igof, et MM. Brown-Boveri et $C^{\text {io }}$ ensuite, ont-ils élé amenés à adopter le système à action au commencement de leur turbine pour la partie à haute pression ct à crécr un type dit mixte ou combiné.

Ces constructeurs emploient même, comme partie à aclion, une roue Curtis, c'est-à-dire une roue possédant ordinairement deux et quelquefois trois étages de vitesse, afin de pouvoir réaliser, dès le début, une très forte chute de pression et amener immédiatement la vapeur dans la pre- mière chambre de la turbine à une pression relativement faible presque égale souvent à la pression atmosphérique. Le pressc-étoupe du côté haute pression se simplifie alors beaucoup et, la température de la vapeur étant suffisamment abaissée par suite de la forte détente, on n'a pas à redoulcr, même dans le cas de fortes surchauffes de la vapeur, des dilatalions dangereuses pouvant déterminer des contacts entre parties fixes el mobiles comme cela peut arriver lorsque, avec une faible détente de la vapeur au début de la turbinc, il se produit de grands changements de température provenant des variations brusques de charge.

D'autre part, la roue à action, en supprimant un grand nombre d'étages de pression du tambour Parsons, permet de réduire sensiblement la poussée suivant l'arbre ainsi que la longueur de la turbine qui est relativement grande dans les turbines entic̀rement à réaction.

L'mploi de la roue Curtis dans la partie haute pression est d'autant plus justifiée, malgré les pertes assez fortes par froltement de la vapeur qu'elle peut occasionner, que la plus grande parlie de la puissance contenue dans la vapcur peut être fournie par le tambour basse pression el qu'il esl donc possible de sacrifier un peu le rendement dans la haute pression pour se rattraper ensuite dans la basse pression.

la Maison Brown-Boveri adopte encore exclusivement le système à réaction toutes les fois que le débit de vapeur est suffisamment considérable pour permettre d'employer des longueurs d'ailettes assez grandes. Ce sera par exemple lo cas des turbincs ulilisant la vapeur à basse pression s'échappant des machines à piston ou des turbines à contre-pression dont la consommation de vapeur est relativcment considérable. On voit ainsi que MM. Brown-Boveri et $\mathrm{C}^{\text {ie }}$ n'hésitent pas à recourir uniquement au tambour à rćaclion toutes les fois que son emploi est praliquement possible et qu'ils ne paraissent disposés à adopter la roue à action que si celle-ci présente des avantages incontestables.

\section{Classification des principaux type de turbines à vapeur}

D'après ce qui précède, il est possible, pensons-nous, d'établir le tableau suivant pour classer les principaux lypes des turbines construites acluellement d'une façon industrielle.

II csl bien difficile de dire quel est le type de lurbine qui paraîl remplir actuellement les mcilleures conditions. Nous avons eu l'occasion de visiter les principales usines de construction de turbines à vapeur de l'Europe et d'interroger lcurs ingénieurs. Presque tous nous ont avoué qu'au point de vue de la consommalion de vapeur notamment, les divers types de turbines des grands constructeurs étaient à l'heure acluelle sensiblement comparables. Ce qui peut fixer le choix de l'acheteur, à notre avis, c'est beaucoup plus la valeur et le sćrieux du constructeur ainsi que les références qu'il peul donner. (Voir tablcaux page suivante.)

\section{EMPLOI DES TURBINES DANS L'INDUSTRIE}

Les avantages des turbines à vapeur sont actuellement bicn connus et l'on ne discute plus la supériorité des turbines sur les machines à piston, nolamment au point de vue encombrement, dépense d'huile, surveillance, entretien êt même consommation de vapcur, surtout dans le cas des très puissantes unités. Les constructeurs des machines à piston eux-mêmes, ont fini par en convenir et la plupart se sont mis à faire des turbines.

Mais les turbines présentent surtout des avantages considérables dans certaines applications qui ne sont pas suffi- 
samment connues des industriels en France. C'est à l'étude sommaire de ces applications que nous voulons nous borner ici, convaincus de l'intérêt considérable qu'elles peuvent présenler dans l'industrie.

\section{Utilisation par les turbines de la vapeur à basse pression}

Il existe un grand nombre de machines à vapeur à piston possédant une marche intermitlente comme, par exemple, les machines d'extraclion, les moteurs de laminoirs, les pilons, etc... sance avec une chule de pression de ro kgr. à I kgr., c'està-dire à la pression atmosphérique, que si la vapeur se détendait de I ligr. à o, $\mathrm{l}$ kgr., pression que l'on obtient très facilement avec les condenseurs actuels.

Plus exactement, si l'on prend $\mathbf{x}$ kgr. de rapeur d'eau saturée et sèche à la pression de $\delta \mathrm{kgr}$. par centimètre carré et qu'on la détende jusqu'à la pression absolue de $\mathrm{I} \mathbf{k g r}$., la quantité de chaleur, théoriquement disponible sera de 82,5 calories et la consommalion théorique de vapeur serait de $7,6 \mathrm{~kg}$. par cheval-hcure.

\section{Turbines à Action}

Un seul étage de pression

Deux étages de pression

Plusieurs étages

de pression

(Le nombre d'étages de pression est variable suivant la puissance).
Un seul étage de vitesse.

Plusieurs étages de vitesse

Un étage de vitesse par étage de pression Plusieurs étages de vitesse par étage de pression

Un étage de vitesse par étage de pression Type Rateau

Plusieurs étages de vitesse par étage de pression

Type Curtis

Plusicurs étages de vitesse par étage de pression pour la haute pression (Type Curtis) Un seul étage de vitesse par étage de pression pour la basse pression (Type Rateau)
(De Laval (Puissances inférieures à 100 HP). (Electra (Puissances inférieures à $50 \mathrm{HP}$ ).

De Laval (de 100 à 300 HIP).

I Electra (de 50 a 1000 IIP.

\section{Rateau.}

Eertikon.

ZoELLY.

arec disqnes

\section{Breguet.}

Dujardin.

Turbines marines.

Rateat.

arec tambour

Curtus. (axe rertical).

A. E. G. faibles puissances (axe horizontal).

Curris (grandes puissances).

A. E. G. (moyennes et grandes puissances).

\section{Turbines à Réaction}

Degré de réaction $1 / 2$ - Turbines Parsoxs

\section{Turbines mixtes ou combinées à action et à réaction}

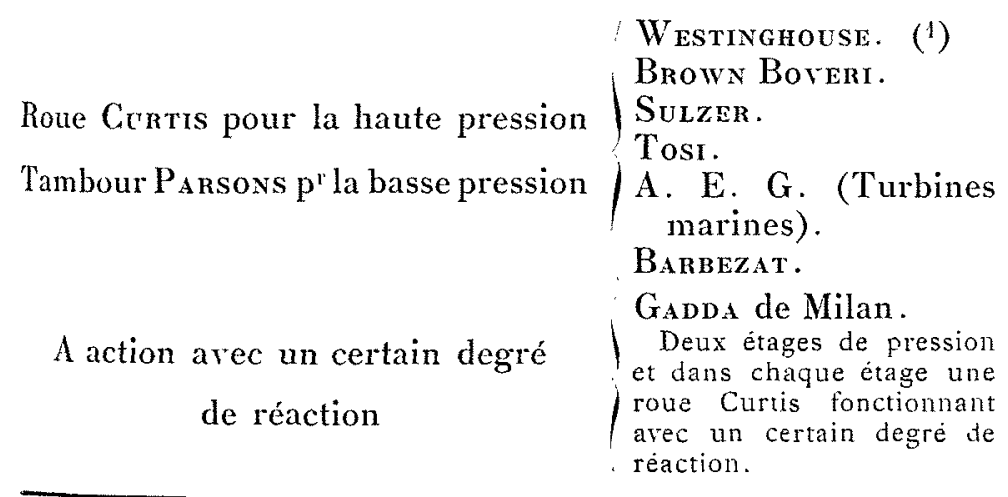

Avec de telles machines, les condenseurs ont un fonctionnement défeclueux et ne permettent pas de réduire la consommation de vapeur de plus de i 5 à $25 \%$ au grand maximum. L'installation des condenseurs étant coûteuse, on préfère souvent faire fonctionncr ces machines à échappement libre.

L'énergie perdue ainsi est considérable et il est facile de s'en rendre compte en remarquant que la puissance que peut fournir la vapeur en sc détendant dépend non de la différence des pressions existant avant et après la détente, mais plutôt du rapport des pressions, de sorte que l'on peut, théoriquement du moins, oblenir à peu près autant de puis-

(i) La Société Wrstranghouse construit, depuis quelque temps, les turbines Rateiv.
Si maintenant, au licu de ne pousser la détente que jusqu'à I $\mathrm{kgr}$., on fait échapper la vapeur dans un condenscur ordinaire pouvant maintenir un vide de $0,1 \mathrm{~kg}$. par centimètre carré, la quantité de chaleur disponible théoriquement sera de 155,5 calories et la consommation théorique de 4,07 kgr. par cheval-heure.

Au lieu de faire échapper à l'air libre la machine à piston, si l'on envoyait sa vapeur dans une excellente machine capable de bien uliliser le vide d'un bon condenseur, il serait possible de récupérer ainsi une puissance importante presque égale à celle de la machine à piston.

Seulement une machine à piston ne conviendrait pas du tout pour remplir ce rôle car clle uliliserait mal le vide du condenseur, la détente de la vapeur ne pouvant pas être complète dans les cylindres à moins de donner à ceux-ci des dimensions inacceptables qui conduiraient d'ailleurs à des pertes par frottement exagérées.

La turbine à vapeur est, au contraire, tout à fait indiquée dans ce cas puisque, avec des dimensions restreintes, elle permet l'écoulcment d'une quantité considérable ou plutôt d'un volume énorme de vapeur et, d'autre part, la partie mobile tournant presque dans le vide, les frollements deviennent très faibles.

La turbine à vapeur fonctionnant à basse pression a d'ailleurs un meilleur rondement que la turbine à hante pression, ce qui est le contraire pour une machine à piston. Ainsi, dans une machine à piston compound, on peut admettre que le rendement, ou rapport da travail effectif sur l'arbre au travail theorique disponible, est pour le cylindre a haute pression de 70 à $80 \%$ et de 30 à $10 \%$ pour le cylindre basse pression, tandis que, dans le cas d'une turbine, 
le rendement serait de 60 à $70 \%$ pour la partie haute pression et de 70 à $80 \%$ pour la partic basse pression.

M. Rateau est le premier à avoir eu l'idće d'utiliser les énormes quantités de vapeur d'échappement perdues ainsi par les machines à marche intermittente pour les employer à actionner des turbines à vapeur.

Afin de rendre régulier le fonctionnement de la turbine à vapeur, malgré les intermittences de marche des machines à piston, M. Rateau a imaginé un accumulateur de vapeur destiné à maintenir sensiblement constante la pression avant la turbine. Gelui-ci, qui n'est autre chose qu'une chaudière à basse pression, est formé en principe d'un grand récipient en tôle garni intérieurement de ferraille ou d'augels remplis d'eau de façon à constituer une masse capable d'absorber la chaleur de la vapeur, de condenser en partie cclle-ci pendant lc fonclionnement des machines à piston et de la restituer ensuile pour la fournir à la turbine lorsque les machines à piston sont arrêtées.

Les Lurbines à basse pression ont pris à l'heure acluelle un grand développement, aussi bien en France qu'à l'étranger, mais il a fallu, pour les fairc admettre, vaincre bien des oppositions. Il est bon de citer à ce sujet le passage suivant cxlrail du Mémoire de M. Rateau sur "Los lurbo-machines multicellulaires et leurs récentes applicalions " (1).

"La première installation ful, comme on le sait, faite en 1902 aux Mines de Bruay avec une pelite turbine de 3 oo chevaux de construction Sauller Harlé, aclionnanl une généralrice à courant conlinu. Ce système s'est ensuite développé à l'élranger beaucoup plus qu'en France, où la chose ne fut pas loul d'abord prise au sérieux. Co n'est que lorsque le succès cul élé conslalé en Angleterre of en Allemagne que l'on se décida à l'adopter dans notre pays, et, depuis lors, les installations se sont mullipliécs arce une grande rapidité, à tel point que nous avons déjà 35 ooo HP en France contre 250 ooo à l'ćtranger $"$.

M. Rateau a montré en outre l'avantage très grand que l'on peut retirer, dans bien des cas, de l'emploi des turbines à basse pression même avec des machines à piston à marche continue.

"Si, en effel, dit M. Rateau dans le même Mémoire, les machines à piston doment de bons rendements dans le fonctionnement à haute pression, il n'en esl pas de même pour la basse pression. Elles ne permettent pas d'uliliser toute la chule de pression créce par le condenseur, car, pour cela, il faudrait domner aux cylindres des dimensions énormes et les pertes par frottements el condensations seraient exagérées. 11 y a donc intérêt à achever la délente de la vapcur dans une turbine. Nous arons fait des installations de ce genre à la Centralc d'Edimbourg et, toul récemment, dans le peignage Allart el Rousscau, à Roubaix. Le bénéfice qu'on retire de lelles combinaisons dépasse ordinairement $20 \%$ et atteint jusqu'à $40 \%$ dans les cas favorables $"$.

(3) Bulletin d'avril rgro de le Société des Ingénieurs Civils de France.
Voici les résultats des essais effectués chez MM. Allart et Rousseau à Roubaix sur une machine compound à obturalcur Corliss et délente Frikarl fonctionnant d'abord seule sur un condenseur W. L. puis combinée avec une turbine à vapeur système Rateau à basse pression actionnant un alternateur triphasé de $450 \mathrm{kw}$. et échappant dans un condenseur W. L.

La MACHINE a piston fONCTIONNe seule (essai du 27 jalvier rg ro) :

Consommation de vapeur on $\mathrm{kgr}$. et par heure mesurée d'après l'alimentation aux chaudières .... 7504

Puissance totale en chevaux indiqués ..........

Consommation de vapeur en kgr. par cheval indiqué et par heure ................. 904

8,3

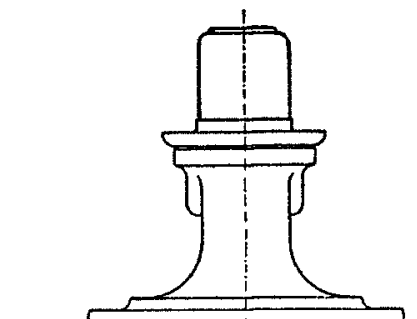

Frg. 7. - Turbine Brown Boveri Parsons utilisant la vapeur d'échappement de machine à piston.

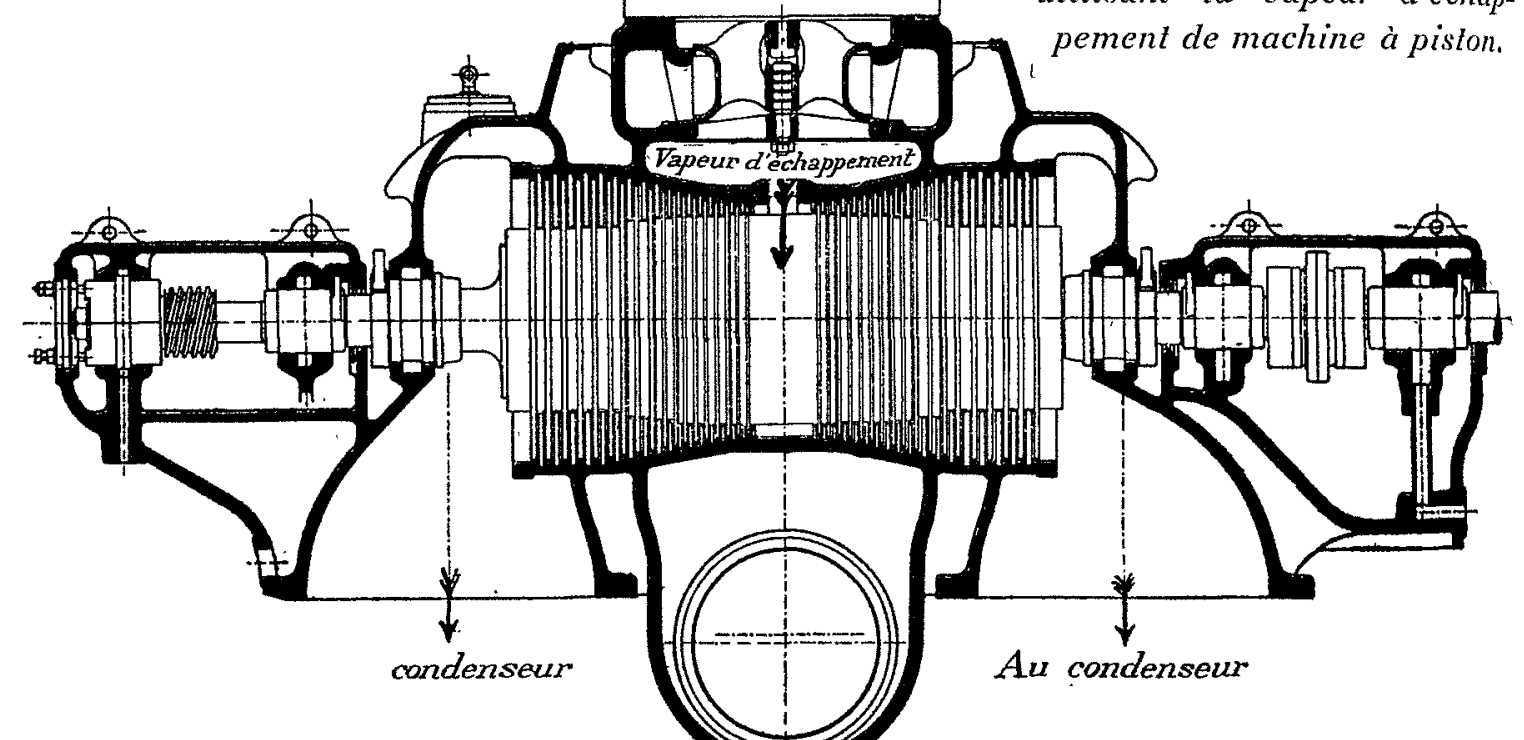

LA MACHINe a PISTON FONGTIONAE AlEC LA TURBINe (essai du ro juin rgro) :

Consommation de vapeur en Kgr. par heure mesuréc d'après l'alimentation des chaudières ...... Puissance en chev. indiqués à la machine à piston Puissance aux bornes de la génératrice, $325 \mathrm{kw}$., soit en chevaux indiqués .............. Puissance totale en chev. indiqués de l'installation Consommalion de vapeur en Kgr. par cheval indiqué et par heure .................. 7250 715

Augmentalion de la puissance de l'installation en chevaux indiqués :

$$
\frac{1318-004}{904}=45,7 \%
$$

\section{Turbines mixtes ou turbines à deux vapeurs}

Lorsque la machine à piston doit s'arrêter pendant long. lemps, l'accumulateur peut devenir insuffisant pour fournir' la vapeur nécessaire au fonclionnement de la turbine qui risque dans ce cas de s'arrêter.

Or, il est souvent indispensable que la turbine puisse continuer à assurer son service normal malgré l'arrêt des machines à piston. 
On peut alors, comme M. Rateau l'avait fait aux Mines de Bruay pour sa première turbine, puiser de la vapeur directcment à la chaudière lorsque la vapeur d'échapjoment vicnt à manquer et la détendre jusqu'à la pression de l'accumulateur de vapeur. Tout revient donc, dans ce cas, à remplacer la machine à piston par un détendeur.

Mais l'utilisation de la vapeur prise directement à la chaudièrc est dans ces conditions très mauvaise et le rendement do l'ensemble devient trop médiocre lorsque les machines à piston sont appelées à subir des arrêts fréquents et prolongés, cas de bien des installations.

Cet inconvénient peut être facilement supprimé car il suffit de remplacer le détendeur par une turbine ordinaire à hale pression qui vient ajouter sa puissance à la turbine basse pression et n'entre en jeu que lorsque la vapeur d'échappement devient insuffisante.

On a ainsi une turbine mixte alimentée par deux vapeurs : de la vapeur fraîche à haute pression et de la vapeur d'échappement à basse pression.

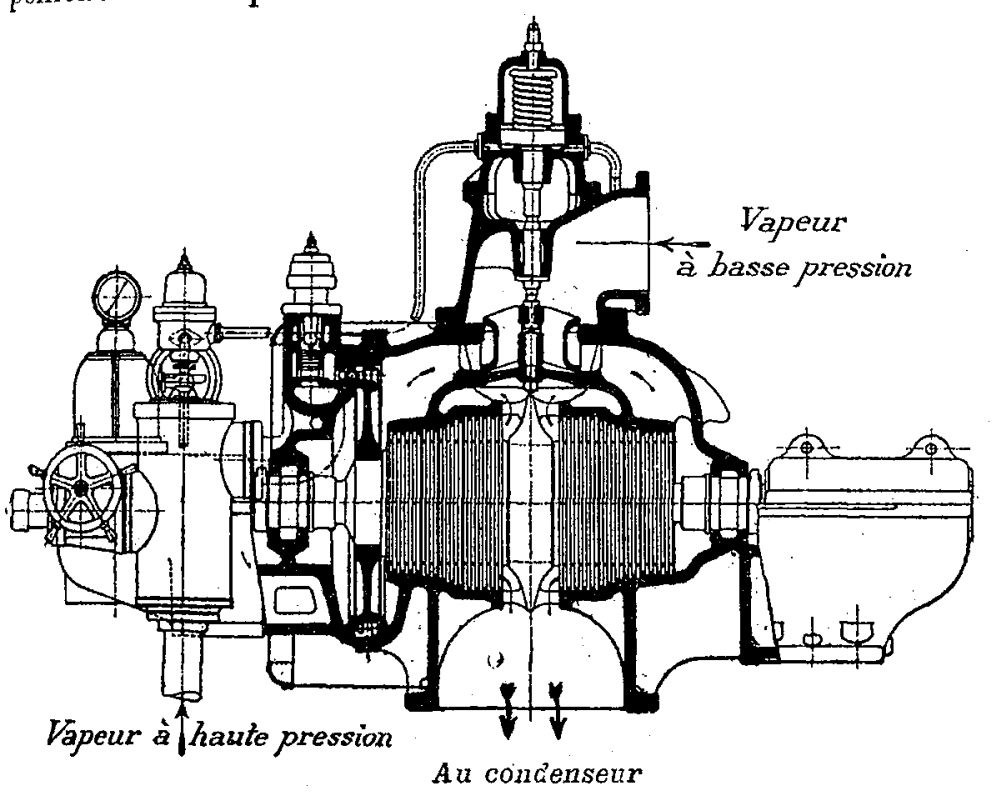

FIG. 8. - Turbine mixte Brown Boveri Parsons.

Un régulateur automatique commande sans difficulté l'ar.rivée de ces deux vapeurs pour maintenir constante la vilesse de la turbine et la pression dans l'accumulateur.

La turbine mixte fonctionnant avec de la vapeur à haute pression ne peut pas cependant avoir un aussi bon rendement qu'une turbine ordinaire. Pour obtenir, en effet, la même puissance avec la vapeur vive, le poids de vapeur consommé n'atteint guère que la moitié environ de celui qui est nécessaire avec la marche à basse pression. La pression absolue baisse alors de près de moitié à l'entrée de la turbine basse pression qui devient mal utilisée.

Dans le but d'éviter cette diminution de rendement, les Ateliers de Construction Oerlikon ont imaginé une turbine mixte, appelée série parallèle, qui fonctionne de la manière suivante :

La partie basse pression de la turbine est divisée en deux régions distinctes l'une de l'autre et pouvant fonctionner indépendamment. Pour séparer ces deux régions, les diaphragmes, c'est-à-dire les disques fixes, possèdent des nervures présentant un jeu très faiblc avec les disques mobiles et empêchant dans chaque étage de pression la vapeur dc passer d'une région dans l'autre.

Lorsque la machine reçoit uniquement de la vapeur d'échappement, les deux régions basse pression fonctionnent en parallèle, mais, si la vapeur d'échappement venant à être supprimée, la turbine doit s'alimenter avcc de la vapeur à haute pression, le débit de vapeur ćlant alors bien déterminé, une scule région de la partie basse pression est mise en service et se trouve disposée en série avec la partie à haute pression. On peut donc par ce moyen mainlenir la pression constante à l'entrée de la partic à basse pression ct oftenir un bon rendement lorsque la turbine reçoit de la vapeur vive.

Cependant, en tenant compte dans les calculs de la baisse de pression qui se produit à l'entrée de la partie basse pression et en établissant la turbine convenablement, il est possible d'obtenir des consommalions de vapeur relativement très réduites sans recourir à aucun artifice entraînant toujours une certaine complication.

Pour le montrer nous croyons intéressant de donner ici les résultats des essais de consommation d'une turbine mixlc ordinaire construite par la Société l'Horme et Buire de Lyon. Ces résultats sont très remarquables, étant donné surtout qu'ils ont pu être obtenus sur une turbine mixte de puissance relativement faible.

Essais de consommation effectués le r3 novembre 1910 par l'Association Lyonnaise des Propriécatres d'Appareus $\wedge$ VAPEur, sur une lurbine mixte (à deux vapeurs), syslème ZoElLy avec régulateur RÁtenv, construile par la Sociélé Horme et Buire et installée aux Mines de La Peronnière.

Caractéristiques de la trubine:

Puissance ....... 500 IIP
Nombre de tours $\ldots .2400$
Condensation par surface.

Cette turbine utilise la vapeur d'échappement d'une matchine d'extraction construite également par les liorges de l'Horme et elle actionne un allernatem de $360 \mathrm{kw} .3$ ooo v. So périodes, de la Société Alsacienne de Construelions Mévaniques.

\section{Résultats des essins}

Marche à basse pression

Puissance fournie aux bornes... .

Puissance fournie au manchon de

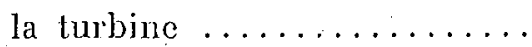
Nombre de tours ........... 2400.

Pression absolue avant la vanne

d'admission .............

Vide au condenscur ..........

Consommation par kilowalts aux bornes ............... Consommation par HP sur Ic manchon d'accouplement .... $3.17 \mathrm{Kw}$. $5{ }_{7}, 87 \mathrm{HP}$.

I,, $30 \mathrm{Kg} \cdot$ par $\mathrm{cm}^{2}$. 92 pour 100 .

I 6,87 г Kg. par heure.

I $1,3:$ hg. par hesure.

\section{Marche à Haute Pression.}

Puissance fournic aux bornes .... 372 Kw.

Puissance fournie au manchon de

la turbine $\ldots \ldots \ldots \ldots \ldots \ldots .552,35 \mathrm{HIP}$.

Pression avant le régulateur .... $8,766 \mathrm{Kg} \cdot \mathrm{par} \mathrm{cm}^{2}$.

Vide ............... g1,2 pour 100.

Consommation par kilowalls aux

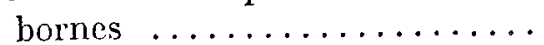

Consommation par IHP sur Ic

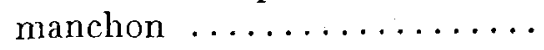
го, $266 \mathrm{Kg}$. par heurc.

6,9 / $\mathrm{Kg}$. par heure.

Les consommations de vapeur ont été calculćes en déterminant la quantité d'eau condensée. 
La puissance de l'alternateur était absorbéc par un rhéostat liquide permettant d'obtenir une marche très constante.

\section{Turbines fonctionnant à contre pression pour permettre le chauffage par la vapeur}

Un très grand nombre d'usines, comme les papetcries, les teintureries, les fabriques de produits chimiques, etc., ulilisent de la vapeur à basse pression pour le chauffage.

Le plus souvent, encore maintenant, on se contente de prendre de la vapeur direclement aux chaudières et de l'abaisser à la pression voulue au moyen de détendeurs.

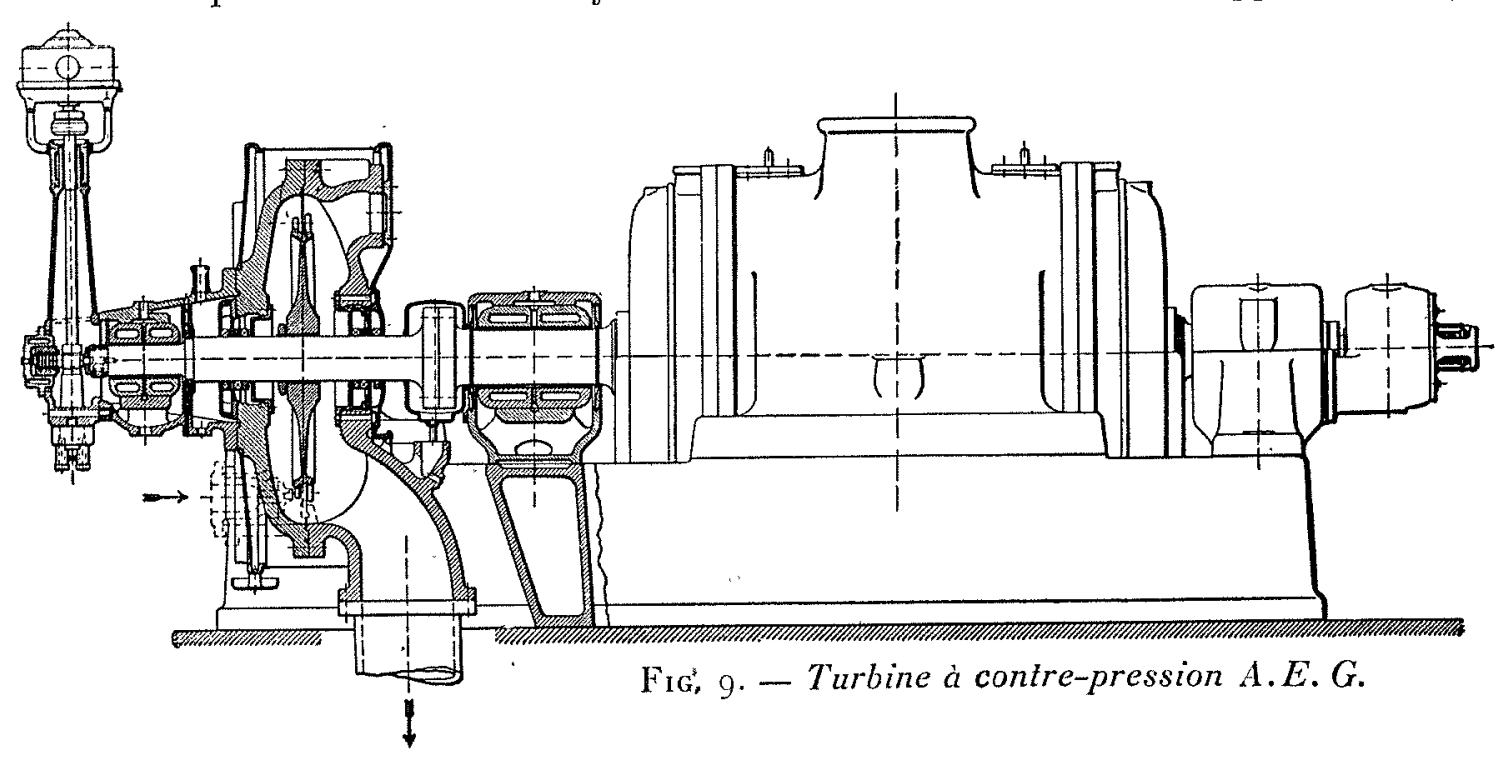

Quclquefois, ccpendant, lo détendeur est remplacé par une machine à vapeur à piston qui peut fournir dans ces conditions une force motrice importante presque gratuitement. Comme une caloric correspond à 426 kilogrammètres, la puissance engendréc par la machine à vapeur, faisant l'oflice de rlćlendeur, ne correspond donc qu'à une diminution très restreinte de la chaleur contenue dans la vapeur que l'on peut employer cnsuite très efficacement pour le chauffage.

Mais, avec les machines à piston, on a ̀̀ craindre un inconvéuient très grave dù à l'huile employée pour le graissage des cylindres qui, entraînée par la vapeur, s̈inlroduit dans les apparcils de chautlage, les encrasse el surtout s'oppose à la transmission de la chaleur en recouvrant les surfaces chaulfantes d'une couche mauvajse conduclrice de la chaleur.

Il exisle bien de bons séparaleurs d'huile permellant d'arrêter celleci à la sortie des machines à piston, mais ils ne parviennent que bien difficilement à purifier complètement la vapeur el dans bien des usines l'entraînement de l'huile a donné lieu à des ennuis sérieux.

Aver la lurbine à vapeur, les pa-

liers, qui nécessitent seuls un graissage, étant actuellement toujours disposés en dehors de la turbine mème, on peul oblenir directement de la vapeur sans trace d'huile. C'est là un avantage très important.

On a vu que pour l'utilisation de la vapeur à basse pression la turbine ćlait bien supérieure à la machine à piston ; il semblerail que dans le cas de l'utilisation de la vapeur à hautc pression la machine à piston puisse enfin reprendre l'avantage tout au moins au point de vue du rendement. Il est incontestable que dans ce cas la machine à piston absorbera moins de vapeur ou plutôt, pour une quantité de va peur donnée, fournira un peu plus de puissance, mais il convient de bien observer que la puissance supplémentaire perduc dans la turbine ne l'est pas entièrement puisqu'elle sert à réchauffer la vapeur qui a précisément le chauffage pour utilisation principale.

D'autre part, la turbine rendant possible une surchauffe plus grande que dans le cas d'une machine à piston, peut laisser échapper de la vapeur à une température plus élevée ct permet ainsi d'éviter une condensation trop abondante dans les conduites à basse pression.

\section{Turbines à prises de vapeur}

Avec les turbines et les mat chines à piston à contrc-pres. sion la puissance produite sc trouve évidemment liéc à la quantilć de vapeur à fournir. Si l'on doil chauffer beaucoup, on peut disposer d'une force motrice importante, mais, si l'on vient à réduirc le chauffage, la puissance de la turbine diminue forcément à moins que l'on fasse échapper à l'air libre l'excès de vapeur que lós

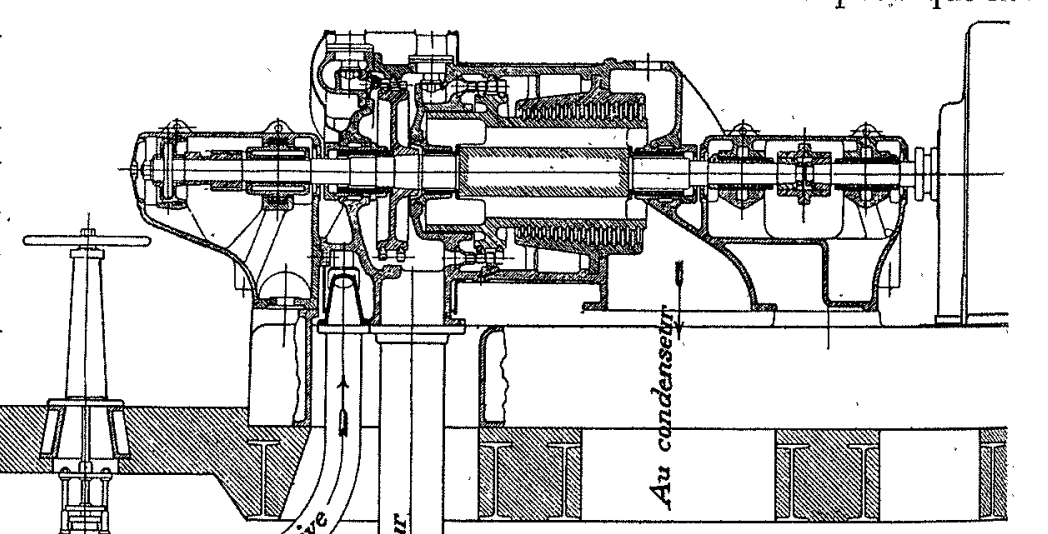

Fig. го. - Turbine Tosi

à prise de vapeur.

apparcils de chauffage ne peuvent absorber.

C'est là un inconvénient, diffificilcment évitable avec lés machines à piston, bien que l'on ait fail quelquefois des prises dans les reccives des machines compound, mais qui n'embarrasse en aucune façon les constructeurs de turbines à vapeur. Si l'apparcil de chauffage n'ulilise pas toute la vapcur qui esl nécessaire pour produire la force motrice, on enverra l'excès de vapeur dans un condenseur après lui avoir fait traverser une turbine à basse pression afin de ne pas laisser perdre son énergie ulilisable.

On a donc été conduit à créer ce que l'on appelle des turbines à prise de vapeur ou à soutirage de vapeur, formées simplement de deux turbines ordinaires calées sur le même 
arbre. L'une ulilise toute la vapeur et délend celle-ci à la pression qui convient aux apparcils de chauffage, tandis fue l'autre reçoit et conduit au condenseur toute la vapeur cn excès qui ne serl pas au chauffage.

Quelles que soient les variations de charge de la turbine et les quantités de vapeur demandées à la prise pour les apparcils de chauffage, la vitesse de la turbine peut être maintenue constante à 2 ou 3 pour ıo près, ainsi que la pression de la vapeur à la prise à 200 ou 300 grammes près.

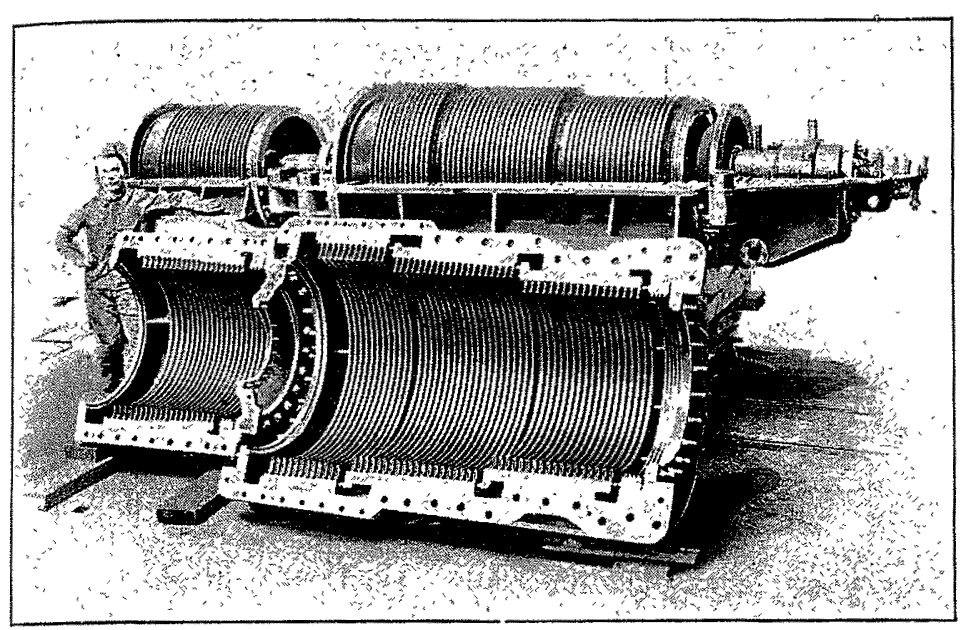

Turbine Bréguet de $2500 \mathrm{HP}$.

Ces turbines à prise de vapeur commencent à être très répandues. La Maison Brown-Boveri en a déjà fourni plus de 25 et la Société A. E. G. une soixantaine. Elles sont surtout employées en Allemagne. En France, il n'en existe pas encore en fonctionnement, mais plusieurs ont été commandées ces temps derniers.

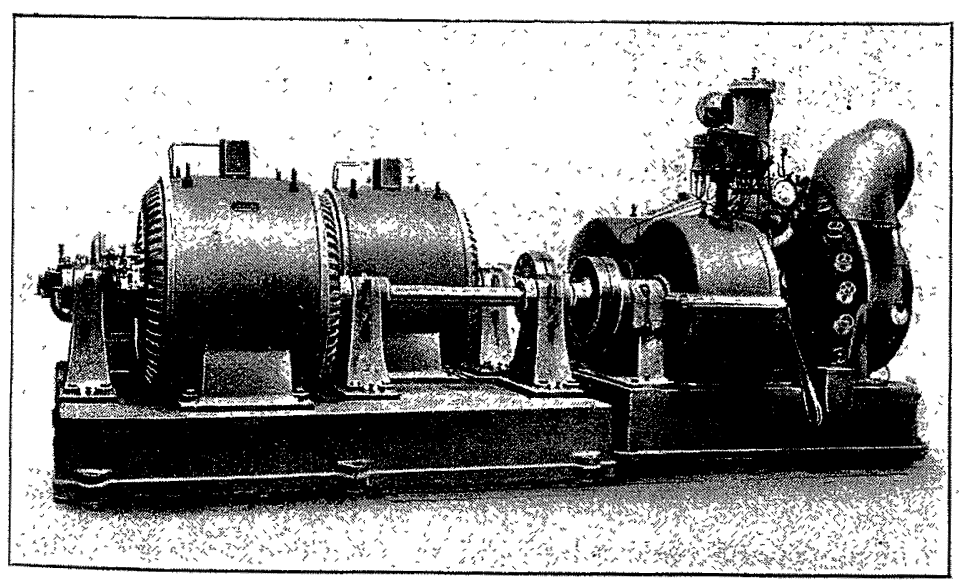

Turbo-alternateur Bréguet (300 kw. 750 t.)

Qu'il nous soit permis pour terminer de rendre ici hommage au mérite de $M$. Rateau qui a contribué d'une manière très efficace au développement des turbines à vapeur et dont la science, qui commence à être justement et universellement appréciée en France, l'est déjà depuis longtemps par les ingénieurs étrangers, comme nous avons pu souvent nous en rendre compte dans les entretiens que nous avons eu l'occasion d'avoir avec eux.

Il est bon de rappeler que M. Rateau a effectué, dès i 895 , à Saint-Etienne, ses expériences sur l'écoulement de la vapeur et que c'est en $1897-1898$ qu'il entreprit la construction de la première turbine multicellulaire à action, sur le principe de laquelle sont basées bien des turbines modernes
L'utilisation de la vapeur d'échappement avec l'emploi de l'accumulateur de M. Rateau a permis de réaliser, surtout dans les mines el la mélallurgie, des économies importantes et acluellement indisculées.

Enfin, les compresseurs et les pompes centrifuges multicellulaires Rateau sont aujourd'hui très répandus dans l'industrie et imités par beaucoup de constructeurs.

On voit donc le rôle très actif joué par M. Rateau dans la conception de toutes les turbo-machines. Et si jamais, dans un avenir qui paraît encore lointain, la turbine à gaz, apportant avec elle la possibilité d'une meilleur utilisation de l'énergie du charbon, vient à détrôner la turbine à vapeur, on trouvera encore sans doute dans cette nouvelle machine, constituant un de ses organes principaux, le compresseur multicellulaire Ratcau. Ce compresscur, MM. I.emale et Armengaud l'ont d'aillcurs déjà utilisé pour faire fonctionner une turbine à pétrole de $400 \mathrm{HP}$, la première furbine à pétrole de grande puissance que l'on ait essayéce.

Paul Bergeon,

Sous-Directeur de l'Institul Electrotechnique de Grenoble

\section{LE CONCOURS D'AMÉNAGEMENT DU RHONE}

Nous donnerons sous peu le rapport du Jury du Concours d'avant-projets pour l'aménagement du Rhòne, que nous avons annoncé dans notre précédent numéro. Il sera reproduit d'après le texte que publiera le prochain Bullelin Lrimestriel de l'Offree des Transports des Chambres de Commerce du Sud-Est, organe officiel des publications du Comité d'organisation du Concours.

Le Président du Jury el rapporteur, M. l'Inspecteur Général des Ponts et Chaussées en rehaite BArLATier de M $\Delta s$ a, d'une manière concise, très nettement dégagé les conclusions du travail considérable que représentent les projets soumis à l'examen de ses collègues. On lira ces conclusions dans notre numéro de Mai. Il ne nous appartient pas de les commenter ; mais, qu'il nous soit pourtant permis de signaler le caractère pratique des vues du Jury.

Depuis longtemps, dans les réunions des Chambres de Commerce, dans les assemblées départementales, dans les Syndicats, les Sociétés Savantes et les Congrès, l'on discourt sur la nécessité de tirer un meilleur parti du fleuve; beaucoup d'idées ont élé remuées et bien des projels mis en avant ; mais la question n'a pas avancé. On dirait que le Rhòne exerce un pouvoir fascinateur sur tous ceux qui l'approchent, mais qu'il repousse de toute la violence de ses flots les présents de l'Industrie dont ses admiratcurs veulent enrichir son cours. De par l'initiative de l'Offuce des Transports, le problème vient d'être à nouveau posé avec un énoncé à la fois précis et général : Jes solutions se sont présentées nombreuses, les unes sous l'aspect de projets giganlesques, d'autres en la forme d'éludes déjà vues, mais comportant une mise au point séricuse, certaines enfin présenlant des moyens qui s'ćcartent beaucoup des voies connues. Du faisceau d'idées ainsi révnies, le Jury du Concours a extrait celles, que, par la possibilité de leur mise en œurre immédiate, il a jugées capables de faire sortir enfin de l'ère des indéterminations celte question du Rhône navigable.

Comme on pourra s'en rendre comple à la lecture da do cument annoncé, le Jury estime que les moyens pratiques de mener à bonne fin la grandiose entreprise considérée, résident en ce programme : 